\title{
An application study of low-dose computed tomography perfusion imaging for evaluation of the efficacy of neoadjuvant chemotherapy for advanced gastric adenocarcinoma
}

\author{
Zongqiong Sun $^{1} \cdot$ Xiaofang Cheng $^{2} \cdot$ Yuxi Ge $^{1} \cdot \operatorname{Lin~Shao~}^{1} \cdot$ Yinghua Xuan $^{3} \cdot{\text { Gen } \operatorname{Yan}^{1}}^{1}$
}

Received: 16 March 2017 / Accepted: 30 August 2017 / Published online: 4 September 2017

(C) The International Gastric Cancer Association and The Japanese Gastric Cancer Association 2017

\begin{abstract}
Background This study used low-dose computed tomography (CT) perfusion imaging technology to evaluate the efficacy of neoadjuvant chemotherapy in patients with advanced gastric adenocarcinoma and to determine whether any of the perfusion parameters could predict tumor response to chemotherapy.

Methods Forty patients with gastric adenocarcinoma (T3-4NxM0) received three cycles of neoadjuvant chemotherapy and low-dose spiral CT perfusion imaging prior to and after the first and third series of chemotherapy. We calculated tissue blood flow (BF) and blood volume (BV) using commercial software. One-way analysis of variance (ANOVA) was used to detect any significant variation of the tested parameters between different times of scanning. Spearman's test was used to evaluate the correlation among perfusion parameters, tumor size and pathological efficacy grade, and clinical response after chemotherapy, respectively. A receiver-operating characteristic analysis was used to determine the optimal diagnostic cutoff value for changes in perfusion parameters and tumor size.
\end{abstract}

Zongqiong Sun and Xiaofang Cheng contributed to this work equally.

Gen Yan

gyan@stu.edu.cn

1 Department of Radiology, Affiliated Hospital, Jiangnan University, Wuxi, People's Republic of China

2 Department of Radiology, Affiliated Brain Hospital of Guangzhou Medical University, Guangzhou Huiai Hospital, Guangzhou, People's Republic of China

3 Department of Basic Medicine, Jiangnan University Medical School, Wuxi, People's Republic of China
Results One-way ANOVA showed significant differences in $\mathrm{BF}$ and $\mathrm{BV}$ values between those before and after chemotherapy $(p<0.01)$. The BF, BV and size reduction rate after three series of chemotherapy were significantly correlated with pathological efficacy grade. BF and BV values after the first and third series of chemotherapy were also significantly correlated with clinical response ( $p<0.01$, respectively). The diagnostic sensitivity and specificity of the BV reduction rate were higher than those of size reduction rate.

Conclusions Low-dose CT perfusion imaging is a valuable tool that permits microcirculation evaluation and therefore can evaluate the efficacy of neoadjuvant chemotherapy in patients with advanced gastric adenocarcinoma.

Keywords Low-dose CT perfusion imaging · Gastric adenocarcinoma $\cdot$ Neoadjuvant chemotherapy $\cdot$ Clinical response $\cdot$ Pathological response

\section{Introduction}

The incidence and mortality rates of gastric cancer have been steadily declining in Western countries in recent decades [1]. However, its incidence and mortality are still ranked first among malignant tumors of the digestive tract in China [2, 3], and the mean survival duration of patients with advanced gastric cancer is less than 1 year [4]. Since Wilke et al. [5] first reported administration of neoadjuvant chemotherapy in the treatment of gastric cancer in 1989, adjuvant therapy for gastric cancer has been extensively studied, and the effect of adjuvant perioperative chemotherapy has been demonstrated in well-designed, multicenter and randomized clinical trials [6]. Previous studies have shown that there is an association between tumor response at anatomical imaging evaluation and histopathological response and survival 
in patients with gastric cancer who are treated with neoadjuvant chemotherapy. However, it still lacks uniform standards to discriminate responders from non-responders; therefore, it is hard to evaluate neoadjuvant chemotherapy's efficacy in gastric cancer treatment [7]. Meanwhile, as it takes time for gross tumor changes to become apparent [7], anatomical imaging may be of limited value in the early assessment of neoadjuvant chemotherapy's efficacy. Low-dose spiral perfusion CT, which can visualize changes in tumor vascular physiology and could thereby potentially be a biomarker for the therapeutic response to chemotherapy [8], has shown great promise in the study of the brain [9], kidney [10], lung [11], etc., by significantly reducing the X-ray dose of CT perfusion scans without compromising the clinical accuracy. In this study, we utilized low-dose spiral CT perfusion imaging to evaluate the efficacy of neoadjuvant chemotherapy in patients with advanced gastric adenocarcinoma and to determine whether any of the perfusion parameters would predict the tumor response to chemotherapy.

\section{Patients and methods}

\section{Study population}

The ethics committee of our institution approved this study, and written informed consent was obtained from all patients after detailed explanations. Forty patients with advanced gastric adenocarcinoma (T3-4NxM0) were prospectively recruited in our hospital from June 2015 to December 2016, including 30 males and 10 females (mean age 61 years; age range 38-70 years). Inclusion criteria consisted of advanced gastric adenocarcinoma with local lymph node metastases and without distant metastases (clinical tumor stage T3 or T4 and $\geq \mathrm{N} 1$ and M0) confirmed by biopsy pathology under endoscopic ultrasonography and patients who completed three cycles of chemotherapy. Exclusion criteria were: allergy to contrast material, impaired renal function and patient unfitness for chemotherapy. All patients received three cycles of neoadjuvant chemotherapy (i.e., ECF chemotherapy regimen: epirubicin $50 \mathrm{mg} / \mathrm{m}^{2}$, intravenous drip, on day 1; cisplatin $60 \mathrm{mg} / \mathrm{m}^{2}$, intravenous drip, on day 1 ; 5 -FU $200 \mathrm{mg} / \mathrm{m}^{2}$, intravenous drip, on day $1-21$, being repeated every 21 days), and tumor surgical resection was performed within 1 week after chemotherapy. The patients were evaluated with a low-dose spiral CT perfusion imaging prior to, after the first series of and after the third series of chemotherapy.

\section{Perfusion CT study protocol}

The stomach is a tubular organ with peristalsis; therefore, the accuracy of the perfusion data and quality of the perfusion image rely on the efficacy of preparations prior to the CT perfusion examination, which included: (1) each patient was required to fast for no less than $8 \mathrm{~h}$ prior to the CT examination; (2) 10-15 min before scanning, hyoscine butylbromide, an anticholinergic reagent, was injected intramuscularly to inhibit gastrointestinal peristalsis. Subjects who had contraindications to this reagent (such as glaucoma, prostatitis, etc.) were excluded. (3) At 5 min before the examination, all subjects were asked to drink $800-1200 \mathrm{ml}$ of warm water to distend the stomach for better visualization of the tumor. (4) An 18-gauge venipuncture needle was placed in the median cubital vein. (5) Participants were instructed in breathing exercises to prolong the breath-holding time to minimize the respiratory rate and amplitude. Furthermore, an abdominal strap was placed around the subject's abdomen to reduce respiratory movement and encourage shallow breathing during the examination.

First, a routine abdominal plain CT scan was performed on a 64-slice spiral CT scanner (Siemens Sensation 64, Germany) with the following parameters: a slice thickness and spacing of $5 \mathrm{~mm}$, tube voltage of $80-100 \mathrm{kV}$, tube current of 60-80 mAs, matrix of $512 \times 512$ pixels and pitch of 0.984:1. Then, a perfusion CT scan was performed based on the images obtained by the CT plain scan; we chose the slice that included the largest tumor area as the center slice; in all, six nearby slices, including the center slice, were selected every $3 \mathrm{~cm}$ on the $Z$-axis. These slices were scanned with a small field of view (FOV) of $4.8 \mathrm{~mm}$ slice thickness, tube voltage of $80-100 \mathrm{kV}$ and tube current of $60-80 \mathrm{mAs}$. A $50 \mathrm{ml}$ bolus of iopamidol (Iopamiro; Bracco; Shanghai, China) containing $300 \mathrm{mg}$ of iodine per $\mathrm{ml}$ was injected using a power injector (MEORAO-Stellant, MEORAO Co., Germany) via an antecubital vein at a rate of $5 \mathrm{ml} / \mathrm{s}$ through an 18-gauge intravenous cannula. Scanning commenced $7 \mathrm{~s}$ after contrast agent injection, and the scan duration was $30 \mathrm{~s}$. Perfusion scan images were reconstructed using an iterative algorithm to decrease the radiation dose and maintain good image quality.

\section{Image interpretation}

Data from the dynamic perfusion CT scan were transmitted to an Advantage Workstation System (ADW4.0, Siemens). The abdominal tumor perfusion protocol in the body CT perfusion 3.0 software (deconvolution method) was then used for data processing. The abdominal aorta was selected as the input artery, and pseudo-color (pcolor) perfusion images were obtained through workstation processing. The region of interest (ROI), which was no less than half of the maximum target lesion area, was drawn as large as possible around the tumor to reduce noise ( $>50$ pixels). Necrotic areas were avoided, and the solid tumor portion was ensured to be as large as possible. Corresponding perfusion parameters, 
namely blood flow (BF) and blood volume (BV), were measured. Measurements of all perfusion parameters were repeated three times, and the averages of the three measurements were shown.

\section{Image evaluation}

Analysis of CT images before and after neoadjuvant chemotherapy was performed in consensus by three experienced radiologists who primarily engaged in gastrointestinal tumor research and were blinded to the clinical findings and surgical or histopathologic results, and they were responsible for recording the image quality (presence or absence of artifacts and stomach cavity filling status), primary tumor size, tumor location and perfusion parameter values. Changes in perfusion parameters as well as primary tumor size between prior to chemotherapy and after chemotherapy were calculated using the following formulae, respectively, in which brackets stand for a tumor perfusion or size measure: (grade 0) and mild change (grade I) were categorized as the pathologically ineffective group.

\section{Statistical analysis}

$\mathrm{BF}$ and $\mathrm{BV}$ values are expressed as mean \pm standard deviation $(\bar{\chi} \pm \mathrm{SD})$. All statistical procedures were run on the Statistical Package for Social Sciences, version 17.0 (SPSS Inc., Chicago, IL, USA). The reference range includes 95\% confidence intervals. One-way analysis of variance (ANOVA) was used to detect any significant variation of the tested parameters between the different times of scanning. The percentages of reduction rates of $\mathrm{BF}, \mathrm{BV}$ and primary tumor size after three series of chemotherapy were correlated with the pathological efficacy grade using Spearman's test. Similarly, Spearman's test was used to evaluate the correlation between $\mathrm{BF}$ and $\mathrm{BV}$ values after the first and third series of chemotherapy and clinical response, respectively. To evaluate the diagnostic accuracy of low-dose CT perfu-

$\%$ Reduction rate $=\frac{[\text { Prior to chemotherapy }]-[\text { After chemotherapy }]}{[\text { Prior to chemotherapy }]} \times 100 \%$.

\section{Clinical evaluation}

The clinical tumor regression was graded based on the standard of Response Evaluation Criteria in Solid Tumors (RECIST, version 1.1), and the specific notations were as follows [12]: complete response (CR), complete disappearance of the tumor; partial response (PR), the maximum diameter of the tumor reduces more than $30 \%$; progressive disease (PD), the maximum diameter of the tumor adds more than $20 \%$ (or $>5 \mathrm{~mm}$ ) or new lesions appear; stable disease (SD), between PR and PD.

\section{Pathological evaluation}

The surgically removed gastric tumor was sent fresh for pathological examination, thinly sliced and promptly fixed in neutral buffered formalin. The tumor was detectable macroscopically; its size was measured, and at least four paraffinembedded sections were stained with hematoxylin and eosin for histological evaluation. All surgical resection specimens were evaluated by experienced pathologists in consensus to assess the pathological response, and the histopathologic tumor regression was semiquantitatively graded based on the General Rules of the Japanese Research Society for Gastric Cancer (JRSGC, 1995) [13]. Patients were divided into two groups: the pathologically ineffective group and pathologically effective group. Patients with moderate change (grade II) and significant change (grade III) were categorized as the pathologically effective group, and patients with no change sion imaging in the prediction of response to neoadjuvant chemotherapy, we performed receiver-operating characteristic (ROC) analysis to determine the optimal diagnostic cutoff value for changes in perfusion parameters as well as tumor size in regard of sensitivity and specificity for predicting pathological response. $p<0.05$ was considered statistically significant.

\section{Results}

Results showed CT perfusion scanning generated small radiation doses (about $5 \mathrm{mSv}$ ), and the image quality met diagnostic requirements. We observed significant decreases $(>50 \%)$ in gastric tumor size in three cases (Figs. 1,2), mild to moderate decreases $(<50 \%)$ in 20 cases and no changes in 17 cases after the first series of chemotherapy. Table 1 shows comparison of CT perfusion parameters before and after chemotherapy for 40 patients with advanced gastric adenocarcinoma. One-way ANOVA showed significant differences in BF and BV values between before chemotherapy and those after the first series of chemotherapy and between before chemotherapy and those after the third series of chemotherapy $(p<0.01)$, while there was no significant difference in $\mathrm{BF}$ and $\mathrm{BV}$ values between those after the first series of chemotherapy and those after three series of chemotherapy $(p>0.05)$. No significant differences were seen for BF and BV values before chemotherapy and after chemotherapy in the normal stomach wall $(p>0.05)$. 

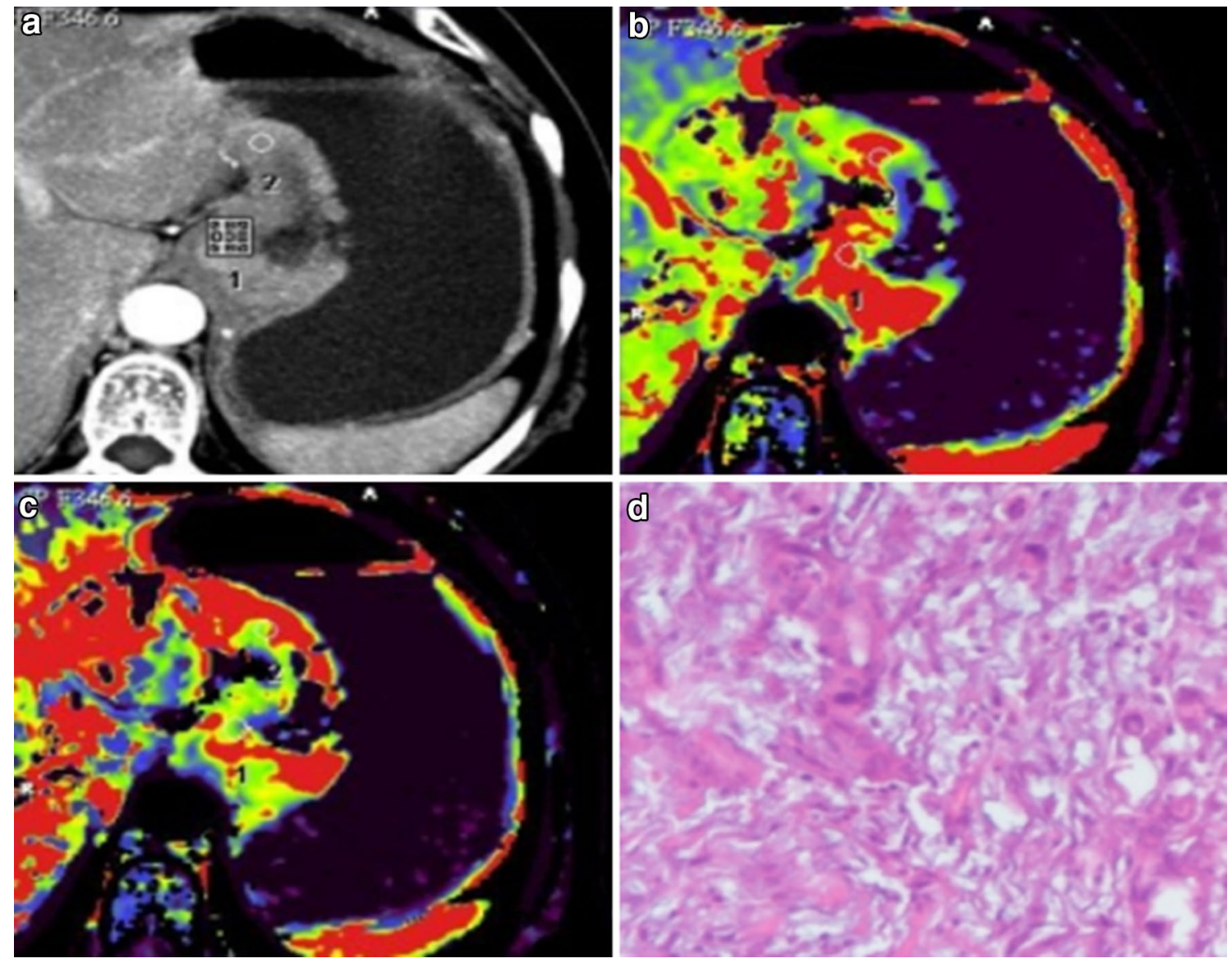

Fig. 1 Images for a 65-year-old male patient with advanced cardia adenocarcinoma (T3N2M0) before neoadjuvant chemotherapy. a The CT perfusion original image shows an irregularly shaped tumor in the cardia and obvious enhancement. b BF and $\mathbf{c}$ BV images show distri-

According to the pathological efficacy evaluation standard proposed by the Japanese Research Society for Gastric Cancer, there were 5 cases with grade 0,20 cases with grade I (15 IA and 5 IB), 11 cases with grade II (Fig. 2d) and 4 case with grade III. Among them, 25 cases were classified as the pathologically ineffective group (grade 0 and grade I), and 15 cases were classified as the pathologically effective group (grade II and grade III). There was a statistically significant difference in $\mathrm{BF}$ and $\mathrm{BV}$ value reduction rates between the pathologically ineffective group and pathologically effective group $(p<0.01)$. The BF reduction rate and $\mathrm{BV}$ reduction rate after three series of chemotherapy were significantly correlated with the pathological efficacy grade $(r=0.536$, $p=0.002$ and $r=0.568, p=0.001$, respectively). The correlation between the BV reduction rate and pathological efficacy grade is shown in Table 2. The size reduction rates after three series of chemotherapy were also significantly correlated with the pathological efficacy grade $(r=0.517$, $p=0.004)$. The correlation between the size reduction rate bution of red and yellow in the tumor area. d A pathological image (hematoxylin and eosin, $\times 100$ ) shows poorly differentiated tubular adenocarcinoma and significant numbers of atypical cancer cells

and pathological efficacy grade is shown in Table 3. The BF and BV values of 40 patients with advanced gastric adenocarcinoma after the first series of chemotherapy were significantly correlated with clinical response $(r=0.615$ and $r=0.637, p<0.01$, respectively). In like manner, the BF and $B V$ values of 40 patients with advanced gastric adenocarcinoma after three series of chemotherapy were significantly correlated with clinical response $(r=0.652$ and $r=0.664, p<0.01$, respectively), and the correlation of clinical response and perfusion parameters $(\mathrm{BF}, \mathrm{BV})$ after the first and third series of chemotherapy is shown in Table 4.

An ROC analysis was used to determine the optimal diagnostic cutoff value for the $\mathrm{BV}$ reduction rate as well as size reduction rate (Fig. 3) after the first series of chemotherapy in patients with advanced gastric adenocarcinoma. The area under the curve (AUC) for BV was 0.833 , and a cutoff value of $34.5 \% \mathrm{BV}$ reduction rate yielded a sensitivity of $73 \%$ and a specificity of $87 \%$ for predicting response after the first series of chemotherapy $(p=0.000)$. Similarly, the AUC for 


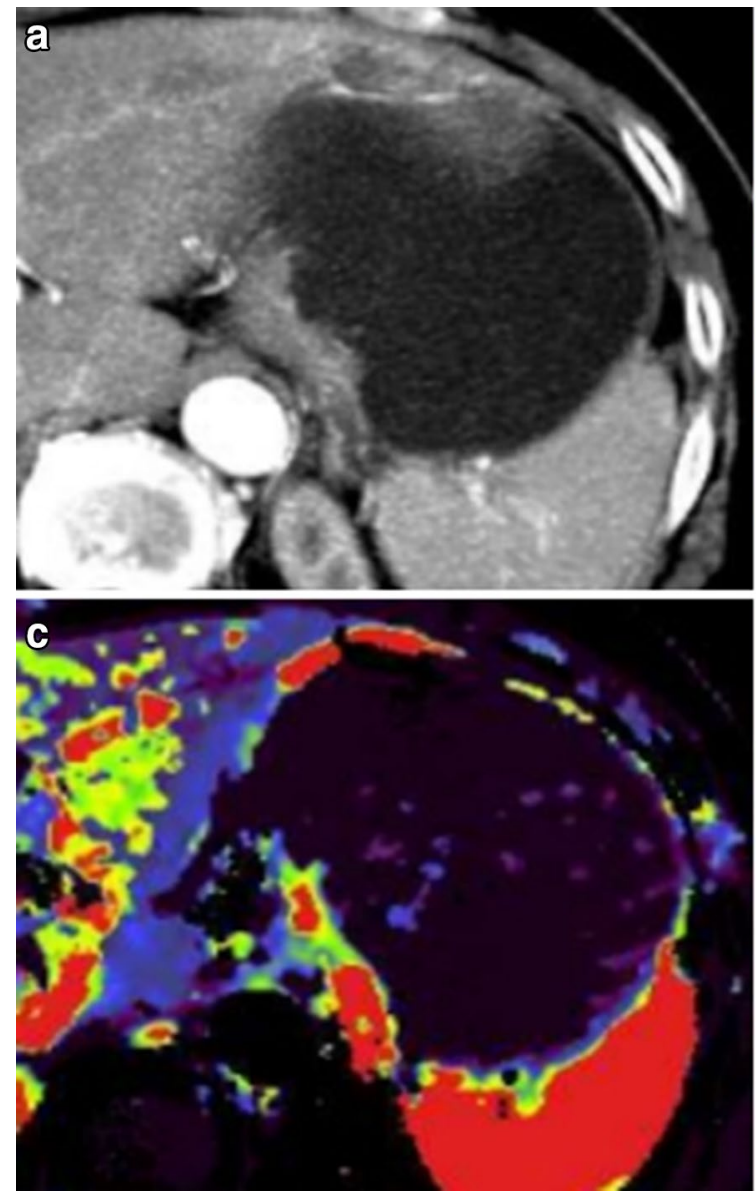

Fig. 2 Images after the first series of neoadjuvant chemotherapy with the ECF regimen for the same patient as in Fig. 1. a-c CT perfusion images show a significantly smaller tumor in the cardia relative to the tumor in Fig. 1. d A pathological image (hematoxylin and eosin,

Table 1 Comparison of BF and BV values prior to chemotherapy, after the first series of chemotherapy and after three series of chemotherapy in 40 patients with advanced gastric adenocarcinoma

\begin{tabular}{lll}
\hline Perfusion parameter & $\mathrm{BF}(\mathrm{ml} / 100 \mathrm{~g} / \mathrm{min})$ & $\mathrm{BV}(\mathrm{ml} / 100 \mathrm{~g})$ \\
\hline Prior to chemotherapy & $95.97 \pm 20.85$ & $16.84 \pm 5.02$ \\
$\begin{array}{l}\text { After first series of chemo- } \\
\text { therapy }\end{array}$ & $76.69 \pm 13.88$ & $11.20 \pm 2.81$ \\
$\begin{array}{l}\text { After three series of chemo- } \\
\quad \text { therapy }\end{array}$ & $70.83 \pm 12.75$ & $10.02 \pm 2.80$ \\
$F$ value & 26.289 & 38.890 \\
$p$ value & $0.000^{*}$ & $0.000^{*}$ \\
\hline
\end{tabular}

Intergroup comparisons were performed by ANOVA among the three groups. There was a significant difference in BF and BV values between before chemotherapy and those after the first series of chemotherapy and between before chemotherapy and those after three series of chemotherapy $(p<0.01)$

*Significant differences
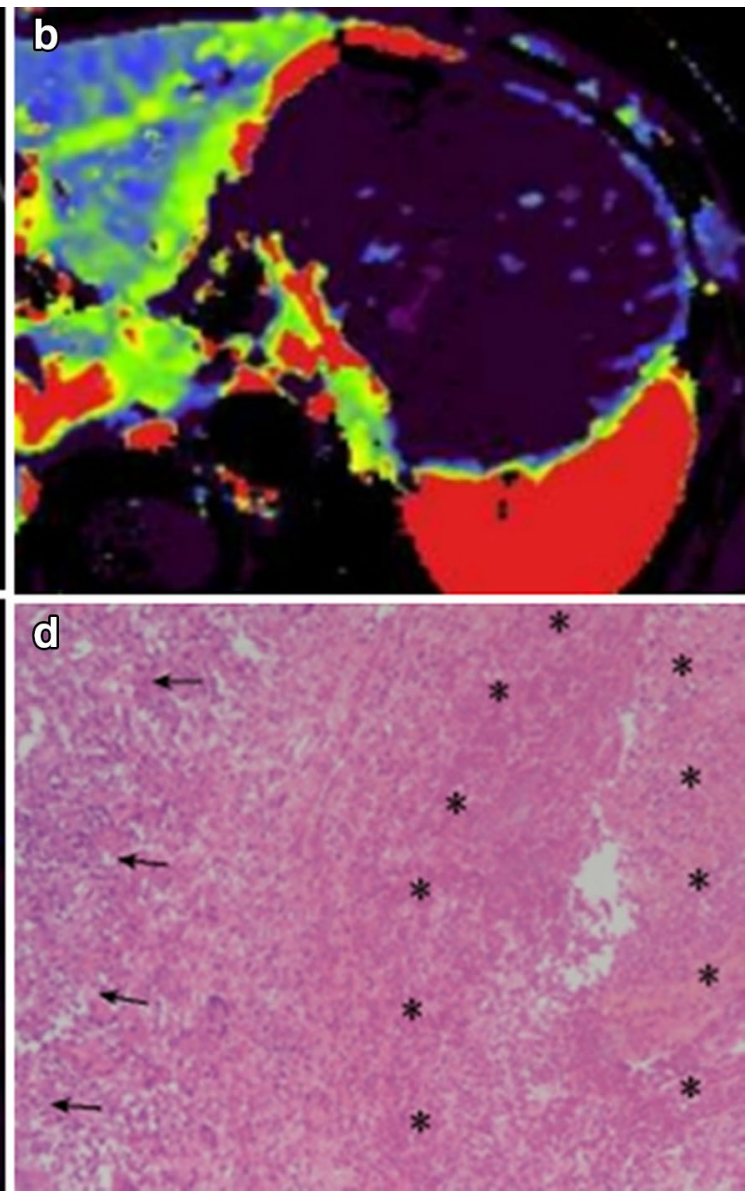

$\times 100$ ) shows necrosis (asterisk) or tumor cell absence in more than two-thirds of the entire focus, but there were cancer cells (upwards arrow) under the microscope. Pathological efficacy evaluation suggested moderate change (grade II)

Table 2 Correlation between the BV reduction rates and pathological response grade in 40 patients with advanced gastric adenocarcinoma $(n=40)$

\begin{tabular}{lrlll}
\hline BV reduction rate (\%) & $<30 \%$ & $30-50 \%$ & $50-70 \%$ & $70-100 \%$ \\
\hline Pathological response grade & & & \\
Grade 0 & 4 & 1 & & \\
Grade IA & 10 & 5 & & \\
Grade IB & 3 & 2 & & 1 \\
Grade II & 1 & 6 & 3 & 2 \\
Grade II & & & 2 & \\
\hline
\end{tabular}

size was 0.755 , and a cutoff value of $32.2 \%$ size reduction rate yielded a sensitivity of $67 \%$ and a specificity of $80 \%$ for predicting response after the first series of chemotherapy $(p=0.025)$. 
Table 3 Correlation between the reduction rate of tumor size and pathological response grade in 40 patients with advanced gastric adenocarcinoma $(n=40)$

\begin{tabular}{|c|c|c|c|c|}
\hline $\begin{array}{l}\text { Reduction rate } \\
\text { of size }(\%)\end{array}$ & $<30 \%$ & $30-50 \%$ & $50-70 \%$ & $70-100 \%$ \\
\hline \multicolumn{5}{|c|}{ Pathological response grade } \\
\hline Grade 0 & 5 & & & \\
\hline Grade IA & 12 & 3 & & \\
\hline Grade IB & 2 & 3 & & \\
\hline Grade II & 4 & 4 & 2 & 1 \\
\hline Grade II & & & 3 & 1 \\
\hline
\end{tabular}

Table 4 Correlation of clinical response and perfusion parameters (BF, BV) after the first and third series of chemotherapy in 40 patients with advanced gastric adenocarcinoma

\begin{tabular}{lccc}
\hline Clinical response & Case $(n)$ & $\mathrm{BF}(\mathrm{ml} / 100 \mathrm{~g} / \mathrm{min})$ & $\mathrm{BV}(\mathrm{ml} / 100 \mathrm{~g})$ \\
\hline $\begin{array}{l}\text { Complete response } \\
\text { After first series }\end{array}$ & 1 & $72.54 \pm 15.41$ & $9.64 \pm 4.21$ \\
After three series & 2 & $69.96 \pm 10.68$ & $8.45 \pm 2.42$ \\
Partial response & & & \\
After first series & 12 & $74.53 \pm 14.71$ & $9.37 \pm 2.34$ \\
After three series & 15 & $69.61 \pm 13.56$ & $8.86 \pm 3.31$ \\
Progressive disease & & & \\
After first series & 1 & $84.26 \pm 13.74$ & $15.57 \pm 2.42$ \\
After three series & 2 & $73.42 \pm 15.84$ & $12.21 \pm 3.24$ \\
Stable disease & & & \\
After first series & 26 & $75.45 \pm 11.67$ & $10.23 \pm 2.27$ \\
After three series & 21 & $70.34 \pm 10.92$ & $10.56 \pm 2.23$ \\
\hline
\end{tabular}

\section{Discussion}

CT perfusion imaging, which is also known as dynamic contrast-enhanced computed tomography (DCE-CT), is an imaging modality that enables noninvasive estimation of tumor vascularization in vivo $[14,15]$. Nevertheless, the consequent heightened radiation exposure of patients who undergo a CT study is still a topic of concern [16]. Currently, various techniques have been developed to reduce the $\mathrm{CT}$ radiation dose. Among them, reducing the tube current and reducing tube voltage are the most practical means of reducing the $\mathrm{CT}$ radiation dose $[17,18]$. However, a reduction in tube current and tube voltage can increase the image noise and degrade image quality and therefore affect the diagnostic accuracy when the filtered back projection (FBP) technique is used [19]. An iterative algorithm for CT was introduced to help reduce the quantum noise associated with FBP reconstruction algorithms $[20,21]$. Previous reports suggested that this technique could provide diagnostic-quality images at 32-65\% lower volume CT dose index (CTDIvol) values than those obtained with the FBP techniques [22].

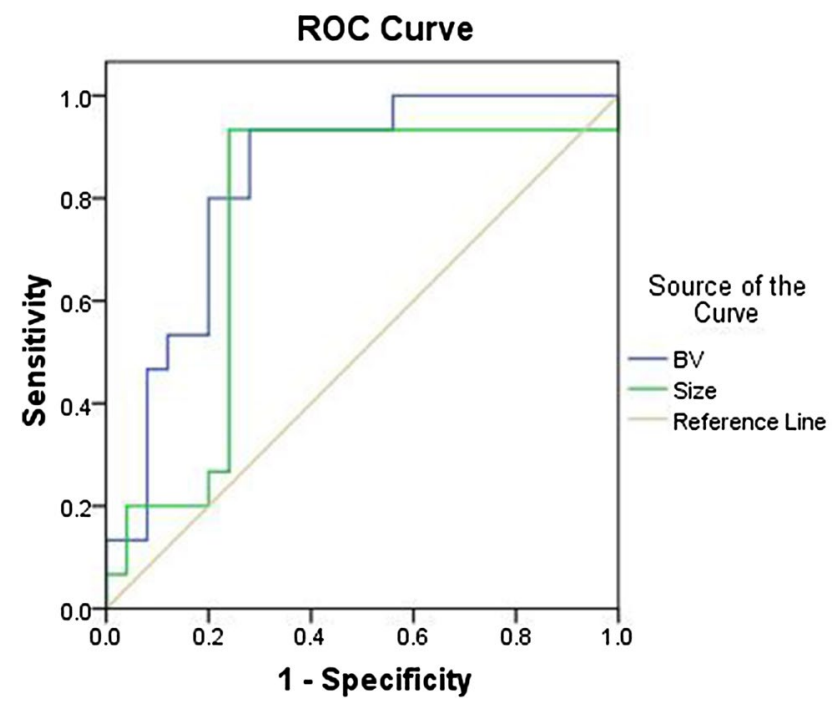

Fig. 3 A graph showing the ROC curve for the BV and size reduction rate. The area under the curve for $\mathrm{BV}$ was 0.833 , and a cutoff value of $34.5 \% \mathrm{BV}$ reduction rate yielded a sensitivity of $73 \%$ and a specificity of $87 \%$ for predicting response after the first series of chemotherapy $(p=0.000)$. The area under the curve for size was 0.755 , and a cutoff value of a $32.2 \%$ reduction rate in size yielded a sensitivity of $67 \%$ and a specificity of $80 \%$ for predicting response after the first series of chemotherapy $(p=0.025)$

Our study found that a combination of low tube voltage $(80-100 \mathrm{kV})$, low tube current $(80 \mathrm{~mA})$ and the iterative reconstruction algorithm technique was adoptable because it can preserve satisfactory image quality while reducing the radiation dose effectively. Meanwhile, we utilized a targeted scanning strategy by choosing a small field of view (SFOV), which meant that the slice containing the largest tumor area was selected as the center slice. With this strategy, it was feasible to reduce the radiation dose and improve the image spatial resolution at the same time. In addition, in consideration of the body size variation, we adjusted the tube voltage $(80-100 \mathrm{kV})$ accordingly to ensure that patients received as little radiation dose as possible with the optimized parameters. In general, the radiation dose of CT perfusion scanning in our study was approximately $5 \mathrm{mSv}$.

Meta-analyses have shown that adjuvant chemotherapy was effective in treating gastric cancer [23]. The 5-year survival rate was reported to be $38 \%$ in patients receiving perioperative adjuvant chemotherapy in comparison with $23 \%$ in those receiving surgery alone [24]. The 2009 NCCN Clinical Practice Guidelines in Gastric Cancer listed neoadjuvant chemotherapy as a recommendation for treatment of advanced gastric cancer. Up till now, many endeavors have been made to evaluate the role of neoadjuvant chemotherapy in the treatment of gastric cancer and explore the optimal strategy for chemotherapy delivery [6]. Some well-designed randomized clinical trials as well as a recently published 
reviews have reached a consensus to propose the ECF regimen as standard care [25].

Several anatomical and molecular imaging modalities have been utilized to predict tumor response to neoadjuvant chemotherapy in gastric cancer. Among them, most of previous studies used anatomical imaging such as routine CT examination and/or EUS, yet the sensitivity and specificity values seemed too low to be used for response assessment in clinical practice [7]. In addition, it takes time before gross tumor changes become apparent [7]. Meanwhile, chemotherapy administration, fibrosis and edema can lead to poorly defined tumor tissues. Thus, it is necessary to evaluate chemotherapy's efficacy from a functional perspective. PET imaging, as a molecular imaging modality, enables us to take direct or indirect measurements of several kinds of pharmacokinetic parameters with high sensitivity by imaging the radioactivity distribution in target organs [26]. Nevertheless, low-resolution, highionization radiation doses and high cost issues have limited the widespread use of PET imaging in clinical practice. CT perfusion imaging is a new imaging modality that permits microcirculation evaluation and therefore can give insight into physiological information about the status of gastric perfusion [27]. In this study, we utilized lowdose spiral CT perfusion imaging to evaluate the efficacy of neoadjuvant chemotherapy in patients with advanced gastric adenocarcinoma and to determine whether any of the perfusion parameters would predict tumor response to chemotherapy.

Our study found that there was evident reduction in primary tumor size of advanced gastric cancer for three cases after the first series of chemotherapy, but there were no visible morphological changes in 17 cases. On the contrary, we observed a significant reduction in the $\mathrm{BF}$ and BV values after the first series of chemotherapy $(p<0.01)$. This means alterations in perfusion parameters preceding to morphological changes can be detected via CT perfusion imaging. Our findings were in accordance with previous longitudinal studies, which demonstrated a significant post-therapeutic reduction in $\mathrm{BV}$ in colorectal cancer [28], lung cancer [29], hepatic tumors [30] and esophageal carcinoma [31]. However, Hansen et al. [32] did not find early changes in blood volume in advanced gastric cancer, although the reduction was significant in absolute numbers after three series of chemotherapy. We correlated the reduction rates of $\mathrm{BF}$ and $\mathrm{BV}$ values after chemotherapy with the pathological efficacy grade by using the Spearman test and found a high correlation between them. We also found that the $\mathrm{BF}$ as well as $\mathrm{BV}$ values after the first and third series of chemotherapy were significantly correlated with clinical response ( $p<0.01$, respectively). Likewise, Hansen et al. [32] found there was a positive correlation between a decrease in tumor permeability $\left(k^{\text {trans }}\right)$ after one series of chemotherapy ( 3 weeks) and treatment response after three series of chemotherapy measured by clinical response, whereas Lee et al. [33] considered that only the volume reduction rate of the primary gastric cancer at CT was significantly correlated to histopathological tumor response. In addition, by performing ROC analysis, we found that the diagnostic sensitivity and specificity of the $\mathrm{BV}$ reduction rate were higher than those of the size reduction rate, and the AUC was larger for the BV reduction rate than for the size reduction rate, indicating that the BV reduction rate possesses better diagnostic performances than the size reduction rate for predicting the pathological response.

There were several limitations to our study. First, our study sample was small for an adequate statistical analysis. Second, the artifact due to respiration was minimized but not avoided, which may limit the quality of images and interfere with the results. Third, we did not evaluate the perfusion parameter and size changes of index metastatic lymph nodes before and after chemotherapy, which might also be useful for evaluating the neoadjuvant chemotherapy efficacy in patients with advanced gastric adenocarcinoma.

In summary, low-dose CT perfusion imaging is a valuable tool that permits microcirculation evaluation and therefore can evaluate the efficacy of neoadjuvant chemotherapy in patients with advanced gastric adenocarcinoma. There are significant changes in BF and BV values after chemotherapy, and the $\mathrm{BF}$ and $\mathrm{BV}$ reduction rates were significantly correlated with the pathological efficacy grade. If the percentage of the $\mathrm{BV}$ reduction rate obtained after the first series of chemotherapy exceeded $34.5 \%$, patients would be categorized as the pathologically effective group with $73 \%$ sensitivity and $87 \%$ specificity. Compared with the authorized RECIST method (tumor size), the CT perfusion parameter (BV) may possess better diagnostic performances for predicting the pathological response.

\section{Compliance with ethical standards}

Conflict of interest The authors declare that they have no conflict of interest, including relevant financial interests, activities, relationships and affiliations.

Funding This work was supported by the Natural Science Foundation of Jiangsu Province (No. BK20151106), Science Foundation of Wuxi Medical Management Center (No. YGZXZ1524) and Wuxi Key Medical Talents (ZDRC032).

Ethical standards All procedures followed were in accordance with the ethical standards of the responsible committee on human experimentation (institutional and national) and with the Helsinki Declaration of 1964 and later versions. Informed consent or a substitute for it was obtained from all patients included in the study. 


\section{References}

1. Lee SJ, Lee WW, Yoon HJ, Lee HY, Lee KH, Kim YH, et al. Regional PET/CT after water gastric inflation for evaluating locoregional disease of gastric cancer. Eur J Radiol. 2013;82:935-42.

2. Wong BC, Lam SK, Wong WM, Chen JS, Zheng TT, Feng RE, et al. Helicobacter pylori eradication to prevent gastric cancer in a high-risk region of China: a randomized controlled trial. JAMA. 2004;291:187-94.

3. Yan SY, Hu Y, Fan JG, Tao GQ, Lu YM, Cai X, et al. Clinicopathologic significance of HER-2/neu protein expression and gene amplification in gastric carcinoma. World J Gastroenterol. 2011;17:1501-6.

4. Bang YJ, Kang YK, Kang WK, Boku N, Chung HC, Chen JS, et al. Phase II study of sunitinib as second-line treatment for advanced gastric cancer. Invest New Drugs. 2011;29:1449-58.

5. Wilke H, Preusser P, Fink U, Gunzer U, Meyer HJ, Meyer J, et al. Preoperative chemotherapy in locally advanced and nonresectable gastric cancer: a phase II study with etoposide, doxorubicin, and cisplatin. J Clin Oncol. 1989;7:1318-26.

6. Li W, Qin J, Sun YH, Liu TS. Neoadjuvant chemotherapy for advanced gastric cancer: a meta-analysis. World J Gastroenterol. 2010;16:5621-8.

7. Kwee RM, Kwee TC. Role of imaging in predicting response to neoadjuvant chemotherapy in gastric cancer. World J Gastroenterol. 2014;20:1650-6.

8. Li ZP, Meng QF, Sun CH, Xu DS, Fan M, Yang XF, et al. Tumor angiogenesis and dynamic $\mathrm{CT}$ in colorectal carcinoma: radiologicpathologic correlation. World J Gastroenterol. 2005;11:1287-91.

9. Othman AE, Brockmann C, Yang Z, Kim C, Afat S, Pjontek R, et al. Impact of image denoising on image quality, quantitative parameters and sensitivity of ultra-low-dose volume perfusion CT imaging. Eur Radiol. 2016;26:167-74.

10. Heuscher DJ, Zhang J, Noo F. Low dose perfusion CT [C]. In: Nuclear science symposium and medical imaging conference, NSS/MIC.2012. IEEE; 2012. p. 3721-5.

11. Yu HY, Zhao SY, Hoffman EA, Wang G. Ultra-low dose lung CT perfusion regularized by a previous scan. Acad Radiol. 2009;16:363-73.

12. Eisenhauer E, Therasse P, Bogaerls J, Schwartz L, Sargent D, Ford $\mathrm{R}$, et al. New response evaluation criteria in solid tumors: revised RECIST guideline (version 1.1). Eur J Cancer. 2009;45:228-47.

13. Ninomiya $Y$, Yanagisawa A, Kato $Y$, Kitagawa T, Ishihara S, Nakajima T, et al. Histological indications of a favorable prognosis with far-advanced gastric carcinomas after preoperative chemotherapy. J Cancer Res Clin Oncol. 1999;125:699-706.

14. Miles KA, Lee TY, Goh V, Klotz E, Cuenod C, Bisdas S, et al. Current status and guidelines for the assessment of tumour vascular support with dynamic contrast-enhanced computed tomography. Eur Radiol. 2012;22:1430-41.

15. Bellomi M, Viotti S, Preda L, D’Andrea G, Bonello L, Petralia G. Perfusion CT in solid body-tumours part II. Clinical applications and future development. Radiol Med (Torino). 2010;115:858-74.

16. Kaul A, Bauer B, Bernhardt J, Nosske D, Veit R. Effective doses to members of the public from the diagnostic application of ionizing radiation in Germany. Eur Radiol. 1997;7:1127-32.

17. Kalra MK, Maher MM, Toth TL, Hamberg LM, Blake MA, Saini S. Strategies for CT radiation dose optimization. Radiology. 2004;230:619-28.

18. Yu MH, Lee JM, Yoon JH, Baek JH, Han JK, Choi BI, et al. Low tube voltage intermediate tube current liver MDCT: sinogram-affirmed iterative reconstruction algorithm for detection of hypervascular hepatocellular carcinoma. Am J Roentgenol. 2013;201:23-32.

19. Patino M, Fuentes JM, Singh S, Hahn PF, Sahani DV. Iterative reconstruction techniques in abdominopelvic CT: technical concepts and clinical implementation. Am J Roentgenol. 2015;205:W19-31.

20. Prakash P, Kalra MK, Kambadakone A, Pien H, Hsieh J, Blake $\mathrm{M}$, et al. Reducing abdominal CT radiation dose with adaptive statistical iterative reconstruction technique. Invest Radiol. 2010;45:202-10.

21. Nakaura T, Nakamura S, Maruyama N, Funama Y, Awai K, Harada K, et al. Low contrast agent and radiation dose protocol for hepatic dynamic CT of thin adults at 256-detector row CT: effect of low tube voltage and hybrid iterative reconstruction algorithm on image quality. Radiology. 2012;264:445-54.

22. Hara AK, Paden RG, Silva AC, Kujak JL, Lawder HJ, Pavlicek W. Iterative reconstruction technique for reducing body radiation dose at CT: feasibility study. Am J Roentgenol. 2009;193:764-71.

23. Panzini I, Gianni L, Fattori PP, Tassinari D, Imola M, Fabbri P et al. Adjuvant chemotherapy in gastric cancer: a meta-analysis of randomized trials and a comparison with previous meta-analyses. Tumori. 2002;88:21-7.

24. Cunningham D, Allum WH, Stenning SP, Thompson JN, Nicolson M, Scarffe JH, et al. Perioperative chemotherapy versus surgery alone for resectable gastroesophageal cancer. N Engl J Med. 2006;355:11-20.

25. Wagner AD, Grothe W, Haerting J, Kleber G, Grothey A, Fleig WE. Chemotherapy in advanced gastric cancer: a systematic review and meta-analysis based on aggregate data. J Clin Oncol. 2006;24:2903-9.

26. Furumoto S, Yanai K. Pharmacokinetics evaluation by PET molecular imaging. Drug Deliv Syst. 2011;26:401-9.

27. Prezzi D, Khan A, Goh V. Perfusion CT imaging of treatment response in oncology. Eur J Radiol. 2015;84:2380-5.

28. Bellomi M, Petralia G, Sonzogni A, Zampino MG, Rocca A. CT perfusion for the monitoring of neoadjuvant chemotherapy and radiation therapy in rectal carcinoma: initial experience. Radiology. 2007;244:486-93.

29. Tacelli N, Santangelo T, Scherpereel A, Duhamel A, Deken V, Klotz E, et al. Perfusion CT allows prediction of therapy response in non-small cell lung cancer treated with conventional and antiangiogenic chemotherapy. Eur Radiol. 2013;23:2127-36.

30. Ma GL, Bai RJ, Jiang HJ, Hao XJ, Dong XP, Li DQ, et al. Early changes of hepatic hemodynamics measured by functional CT perfusion in a rabbit model of liver tumor. Hepatobiliary Pancreat. Dis Int. 2012;11:407-11.

31. Djuric-Stefanovic A, Micev M, Stojanovic-Rundic S, Pesko P, Saranovic DJ. Absolute CT perfusion parameter values after the neoadjuvant chemoradiotherapy of the squamous cell esophageal carcinoma correlate with the histopathologic tumor regression grade. Eur J Radiol. 2015;84:2477-84.

32. Hansen ML, Fallentin E, Lauridsen C, Law I, Federspiel B, Baksgaard L, et al. Computed tomography (CT) perfusion as an early predictive marker for treatment response to neoadjuvant chemotherapy in gastroesophageal junction cancer and gastric cancer-a prospective study. PLoS ONE. 2014;9:e97605.

33. Lee SM, Kim SH, Lee JM, Im SA, Bang YJ, Kim WH, et al. Usefulness of CT volumetry for primary gastric lesions in predicting pathologic response to neoadjuvant chemotherapy in advanced gastric cancer. Abdom Imaging. 2009;34:430-40. 